\title{
Open Source Software Tools for Sequential Analysis and Comparison of Heart Rate Variability in Large Cohort Studies
}

\section{Anurak THUNGTONG}

\author{
School of Engineering and Technology, Walailak University, Nakhon Si Thammarat 80160, Thailand
}

(Corresponding author's e-mail: anurak.th@wu.ac.th)

Received: 8 June 2020, Revised: 13 January 2021, Accepted: 18 February 2021

\begin{abstract}
Heart rate variability (HRV) is commonly used to assess the function of the autonomic nervous system, which is linked to diseases such as cardiovascular disease, diabetes, hypertension, respiratory diseases, and stress. Many studies of the relationship between these diseases and HRV indices have been reported. Generally, the computation of HRV indices is relatively complicated. Moreover, recent researches regarding HRV have employed increasing numbers of electrocardiogram records. Thus, the computation and data processing required are even more complex. Therefore, we propose computer programs for visualizing and analyzing HRV. The proposed programs are developed under MATLAB GUIDE and are available as open source software tools for researchers to develop or modify. We evaluate the programs with MIT-BIH database. The results show that the proposed software tools facilitates the computation of HRV in batch processing mode and the visualization of all of the details, as well as the properties and trends, of HRV indices over long successive epochs. Especially, the software allows us to divide signals into groups for comparing HRV indices. Therefore, the tools are useful for researchers who deal with large cohort ECG signals.
\end{abstract}

Keywords: HRV computation, ECG analysis, Software applications, MATLAB, Signal visualization

\section{Introduction}

The autonomic nervous system (ANS) is a control system that acts largely unconsciously and regulates bodily functions such as the heart rate, digestion, respiratory rate, body temperature, pupillary response, urination, and sexual arousal. ANS is composed of the parasympathetic nervous system (PNS) and the sympathetic nervous system (SNS). It is known that ANS function is related to several cardiac diseases; for example, coronary heart disease, sudden cardiac death, cardiac arrhythmia, heart failure, diabetes, and hypertension [1,2]. Heart rate variability (HRV), the variability of intervals between consecutive heartbeats, is a commonly-used tool for assessing the functional ability of ANS. Increased SNS or decreased PNS activity results in increasing heart rate and decreasing HRV. On the other hand, decreased SNS activity or increased PNS activity causes low heart rate but high HRV. As a result, the degree of variability in heart rate provides information about the functioning of the nervous control over heart rate and the heart's ability to respond [3]. Many studies have reported that HRV is related to some cardiac diseases. For example, decreased HRV during daytime is related to increased cardiovascular risk, such as stroke [4]. Beat to beat variability during deep breathing decreases in diabetic patients before clinical symptoms become evident [3]. It was reported that HRV decreases with recent myocardial infarction [5]. HRV increases after resistance training in individuals with chronic obstructive pulmonary disease [6]. Therefore, HRV is a reliable tool that reflects physiological factors modulating the normal rhythm of the heart.

HRV can be quantified using multiple methods, each reflecting different characteristics of heart rate. Generally, the HRV computation methods proposed in the literature can be divided into 4 main 
http://wjst.wu.ac.th

methods based on domains of signal processing technique used to compute HRV, which are: time domain, frequency domain, time-frequency domain, and nonlinear method. Computer programming has been used to assist in calculating HRV, since it requires computational and signal processing skills. Usually, the calculation starts from pre-processing the ECG signal, detecting R peaks, and computing the $\mathrm{RR}$ interval. The RR interval signal is then used as the input for HRV computation. Several computer programs have been proposed to facilitate HRV analysis, both for commercial and non-commercial use. Some examples are POLYAN [7], ECGLab [8], RHRV [9], a program designed for computing HRV, Twave alternant, and heart rate turbulence [10], Kardia [11], Kubios [12], and HRVanalysis [13]. ECGLab is a MATLAB toolbox that performs $\mathrm{R}$ peak detection and HRV analysis from raw ECG recordings. POLYAN is another MATLAB-based software designed for analyzing several simultaneous signals stored in a hierarchical database. Kardia can analyze multiple datasets simultaneously, calculate average statistics across subjects, and generate analytic spreadsheets that can be used for further statistical analysis. RHRV is designed based on R language with features of statistical calculation. Kubios is a welldesigned graphical user interface with HRV calculation in time domain, frequency domain, and nonlinear methods. Comparison between these software tools has been made [14]. In general, these software tools are designed for different purposes, and have both strengths and weaknesses depending on user requirements.

In this study, we propose two open source MATLAB software tools, one for computing HRV in batch processing mode (HRVmass), and another one for viewing and comparing the results (HRViewer), both with easy-to-use graphic user interfaces. The software HRVmass is designed to detect R peak and compute HRV along successive epochs over the entire recording using several HRV functions. The algorithm for detecting $\mathrm{R}$ peak and computing HRV can be customized. The software HRViewer has been created for viewing details of HRV computation output and providing tools for comparing HRV between groups of cohorts. The ECG signal and detected R peaks can also be viewed. The software HRVmass and HRViewer have been developed using MATLAB. The source files (.m files and GUI) are available for download and modification at https://www.mathworks.com/matlabcentral/fileexchange/73609-hrvmass and ../73610-hrviewer, respectively. The purpose of our softwares is to facilitate users to see the details, an overall view, and a statistical summary of HRV from a single epoch to multiple recording in the same window.

\section{Computing HRV}

The proposed software for computing HRV, namely, HRVmass, was designed to compute HRV in batch processing mode. Data can be imported to HRVmass software as raw ECG or R peak files. The Input data format panel provides radio buttons for selecting types of input files. Accepted $\mathrm{R}$ peak files must be in text format and include a column of timestamps (in seconds) where R peaks occur. For the ECG file, the software takes European Data Format (EDF), which is common for physiological data. The EDF file comes with all important information for reconstructing the raw ECG signal, such as sampling rate, data length, channel names, etc. Loading R peak or ECG files can be done easily by clicking on File -> Add files menu. Then, one or more files can be selected from the file browsing window. The chosen files are then shown in the Files panel.

$R$ peak detection

The automated $\mathrm{R}$ peak detection method used in this software is based on discrete wavelet transform, proposed previously [15]. Briefly, the algorithm attempts to find an optimal way to preprocess the raw ECG signal and the best threshold to distinguish R peaks from other peaks. First, several discrete wavelet reconstruction schemes are performed on the ECG signal using multiple combinations of wavelet detail coefficients. Then, we plot the probability density function for each reconstructed signal. It is assumed that the probability density functions of the reconstructed signals reveal mixed distributions, each belonging to different signal sources such as baseline ripple and $\mathrm{R}$ peaks. The degree of overlapping is then quantified as the "antimode", or the amplitude of the probability density function at the local minimum point found between two main distributions. We also compute the short-term variability of RR 
http://wjst.wu.ac.th

interval of the reconstructed signal. Then, we select the reconstructed signal such that its degree of overlapping plus the short-term variability of RR interval is minimal. Finally, the threshold for separating $\mathrm{R}$ peaks from the baseline and other waves of the selected reconstructed signal is its local minimum point between two main distributions.

\section{Time domain methods}

Several HRV indices had been suggested by the Task Force of the European Society of Cardiology and the North American Society of Pacing and Electrophysiology [16]. Time domain methods are simple ways for computing HRV using RR interval or normal to normal (NN) interval time series. Generally, time domain methods are calculated from the entire recording, typically 24 hour, and represent short to long term variation in RR interval. However, in this software, we focus on sequential analysis of HRV. Therefore, we compute HRV in 5 minutes segments which reflect only short-term variation of RR interval. The two elementary statistical indices are the mean and standard deviation of RR interval (MNN and SDNN, respectively). Other measures of high frequency variability are the root mean square of successive differences of RR interval (RMSSD) and pNN50, which is the percentage of number of successive RR intervals differing by more than 50 milli-seconds.

In addition, there are geometric indices which are computed from a histogram of RR intervals. Suggested bin width used to convert NN interval time series to the histogram is $1 / 128$ seconds. Then, the $\mathrm{HRV}$ triangular index is calculated from total number of RR intervals divided by the height of the histogram. Another geometric index is the triangular interpolation of RR interval histogram (TINN). The calculation of TINN starts from fitting the histogram with a triangle using minimum square difference method. Then, TINN index is measured from the base width of the triangle. Both indices express overall $\mathrm{HRV}$ and are influenced by the lower frequency component of the RR interval time series.

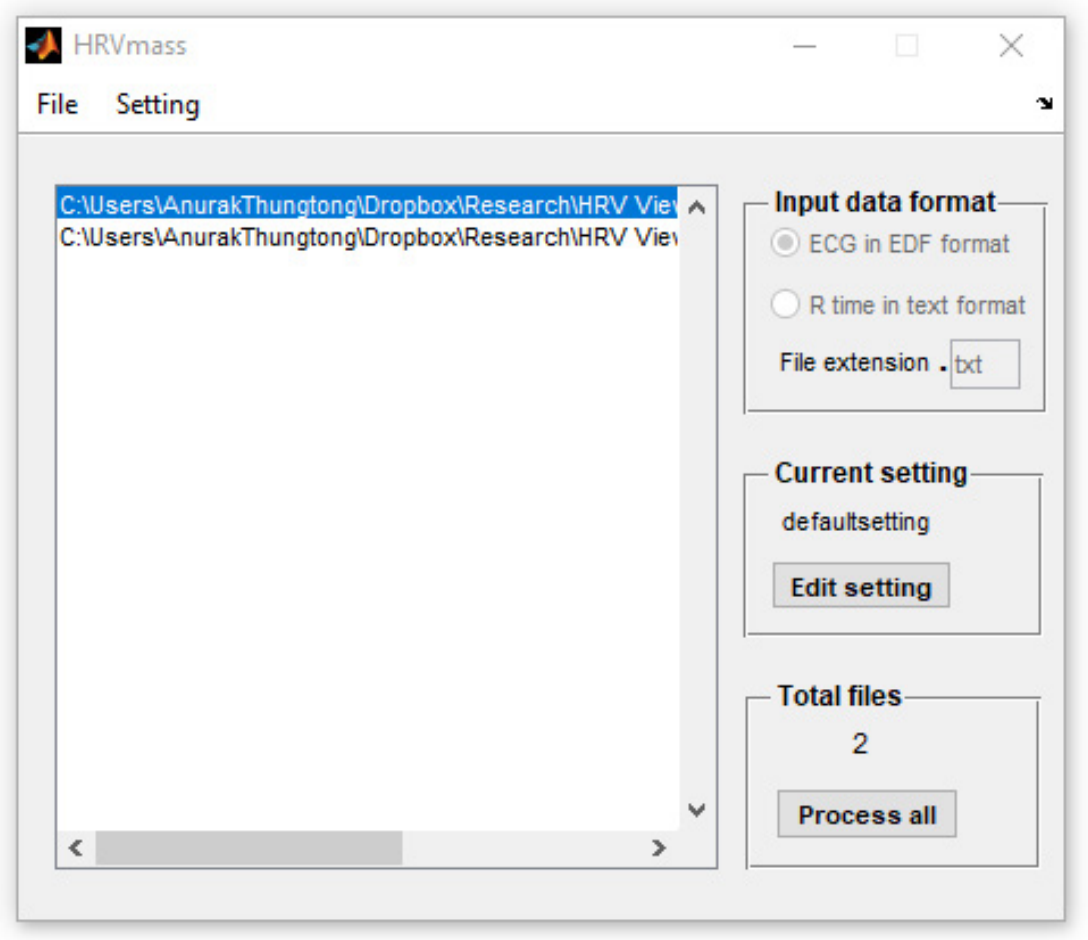

Figure 1 Front view of HRVmass software. 
http://wjst.wu.ac.th

\section{Frequency domain methods}

Frequency domain analysis of RR intervals reveals how variance is distributed as a function of frequency. Calculating HRV in frequency domain requires estimating of the power spectrum of RR intervals. In this study, we employ the Lomb-Scargle method to compute periodogram (power spectral density) of the non-uniform sampled RR interval time series in 5 minutes segments [17]. The power spectral density is divided into very low frequency band (VLF), low frequency band (LF), and high frequency band (HF). The frequency limits of each band for computing HRV in normal human ECG, as suggested in [16], are: $0-0.04 \mathrm{~Hz}$ (VLF), $0.04-0.15 \mathrm{~Hz}$ (LF), and $0.15-0.4 \mathrm{~Hz}(\mathrm{HF})$. HRV indices computed in this software include:

LFP: total power in LF band,

HFP: total power in HF band, and

LHR: power ratio LFP/HFP.

LFP and HFP measure variance of RR intervals in LF and HF bands, respectively. Power in LF and HF bands can also be measured in normalized units (nu.) which represent the relative values of each power component in proportion to the total power minus the VLFP component, where VLFP is the power in VLF band. LFnu and HFnu are defined as:

LFnu $=$ LFP $/($ Total power - VLFP $) \times 100 \%$,

$\mathrm{HFnu}=\mathrm{HFP} /($ Total power - VLFP $) \times 100 \%$.

With normalization, the change in total power has less effect on the values of LF and HF components.

\section{Nonlinear methods}

Several nonlinear measures have been proposed to quantify variability in RR interval signals. In this software, we implement Poincaré plot, approximate entropy (ApEn), sample entropy (SampEn), and detrended fluctuation analysis (DFA).

The Poincaré plot is a scatter plot of $\mathrm{RR}_{\mathrm{i}+1}$ as a function of $\mathrm{RR}_{\mathrm{i}}$; hence, the plot illustrates correlation between current and the next RR interval [18]. The correlation is quantified by fitting the plot with an ellipse aligned along the line of identity. The width of the ellipse, defined as the standard deviation of the points perpendicular to the line of identity, represents short term variability (SD1). The length of the ellipse, defined as the standard deviation of the points along with the line of identity, represents long term variability (SD2). The SDratio (SD1 / SD2) is also calculated.

ApEn and SampEn are used to measure the complexity of RR intervals [19,20]. The RR interval signal with repetitive patterns has a relatively small ApEn and SampEn. The algorithms depend on two parameters: $m$, the embedding dimension (default $=2$ ), and $r$, the tolerance (default $=0.2 \mathrm{SDNN}$ ). The source code for computing ApEn and SampEn is from the copyrighted files of Kijoon Lee [21].

DFA quantifies roughness of RR interval time series by measuring the RR fluctuation at different window widths [22]. The degree of fluctuation is described by two parameters: $\alpha_{1}$ and $\alpha_{2}$ which describe short term and long term fluctuation, respectively. The window width for $\alpha_{1}$ is in the range of 4 - 16 beats, while the window width for $\alpha_{2}$ is in the range of $16-64$ beats. The MATLAB code used in this software is modified from the copyrighted file of Guan Wenye [23].

\section{Additional HRV methods}

Users may add code in the file name processfile.m to compute more HRV methods. Necessary suggestion is already embedded in the file. 
http://wjst.wu.ac.th

\section{Customization}

It is optional to change some basic settings before processing the files using setting window, shown in Figure 2. Here, for example, the ECG channel name, frequency of the notch filter, the segment length, and parameters for HRV methods can be customized before processing the signal and the sequential analyzing of HRV. By default, the output HRV index is saved in the same folder as the ECG or R time file in . mat format. This file will be used later for viewing and comparing HRV results using the software HRViewer, presented in the next section. However, the .txt or .xls file can also be exported if they are selected in the setting window. If the input signal used for computing HRV is raw ECG, the R time stamp is also saved in .txt file for viewing and analyzing. Finally, all settings can be saved for later use.

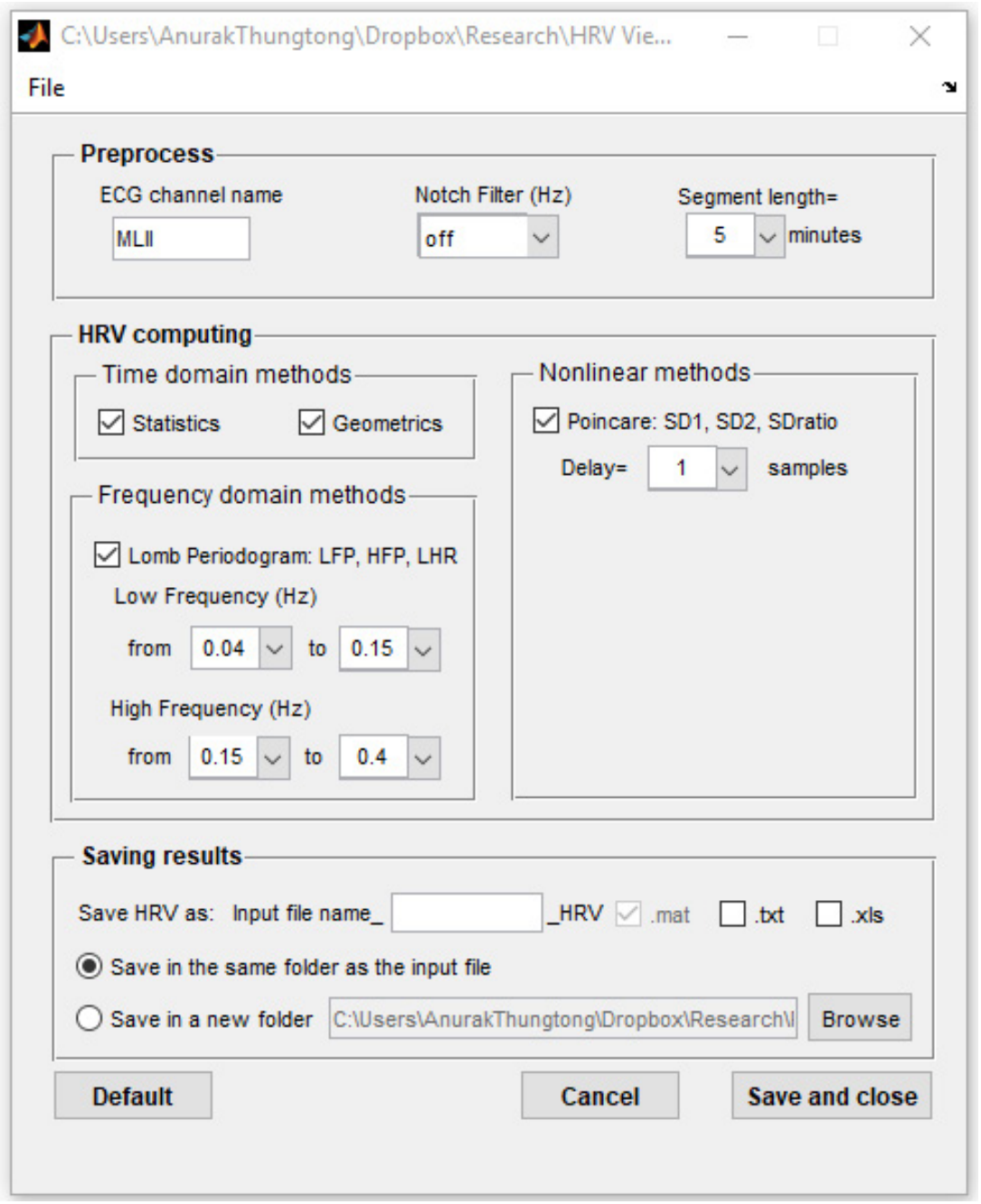

Figure 2 Setting window for customizing HRV parameters.

\section{Validating HRV indices}

The HRVmass software has been validated with PhysioNet Cardiovascular Signal Toolbox [24], a well-documented open-source software designed for validation and standardization in the clinical and scientific community. We use 24 segments with 5 minutes length of RR interval time series of record 100, 
http://wjst.wu.ac.th

101, 102, and 103 from MIT-BIH arrhythmia database [25] for computing sample HRV indices. Table 1 shows average percent difference between HRV indices computed from HRVmass and PhysioNet toolbox. The mean percent difference of each index is defined as:

$D=\frac{1}{N} \sum_{i=1}^{N} \frac{\left|x_{i}-y_{i}\right|}{y_{i}} \times 100 \%$

where $x_{i}$ is the HRV index computed from RR interval segment $i$ using HRVmass, $y_{i}$ is the HRV index computed from RR interval segment $i$ using PhysioNet toolbox, and $\mathrm{N}$ is the total number of segments. Unfortunately, not all indices are included in PhysioNet; therefore, only some indices are validated.

Table 1 Mean percent difference of some HRV indices computed using HRVmass and PhysioNet toolbox. Some indices are not compared because they do not exist in PhysioNet toolbox.

\begin{tabular}{lccccccc}
\hline \multirow{2}{*}{ Time Domain } & MNN & SDNN & RMSSD & pNN50 & TRIAN & TINN \\
& 0.019 & 0.202 & 0.196 & - & - & - \\
\hline \multirow{2}{*}{ Frequency } & Domain & LFP & HFP & LHR & LFnu & HFnu & \\
& 432.948 & 59.30 & 241.805 & - & - & & \\
\hline \multirow{2}{*}{ Nonlinear Methods } & SD1 & SD2 & SDR & ApEn & SampEn & Alpha1 & Alpha2 \\
& 5.029 & 0.409 & 5.328 & 6.902 & 18.597 & - & - \\
\hline
\end{tabular}

Based on the results shown in the table, the mean percent difference of time domain method is no more than 1 percent. Frequency domain methods show relatively high percent difference. The mean percent differences of nonlinear methods are less than 10 percent, except for sample entropy. High percent difference is normal due to different offset and how each software deals with outlier in preprocessing of the data. These results show that it is not suitable to compare HRV indices among different studies, but it can still provide valid comparison within a study.

\section{Viewing HRV}

Consider a situation in which a researcher would like to view the RR interval and HRV time series of some subjects to see how the signals change over time or change corresponding to an event under study. To do so, the researcher starts from recording ECG signals from all subjects. Then, the researcher computes HRV of all ECG files using the software HRVmass. The software HRVmass produces several HRV.mat output files, each containing time series of RR intervals and HRV indices measured from the raw ECG of each subject. For simulation purposes, we employ 4 ECG records from MIT-BIH arrhythmia database as a sample run. The raw ECG signals in EDF format are loaded to the HRVmass software. All settings are set to the default value. The $\mathrm{R}$ peak detection and HRV calculation are run in batch processing mode.

The software HRViewer has been developed for viewing HRV using HRV.mat files, which are the output files from the software HRVmass. The HRV.mat files are imported to the software HRViewer first by clicking on menu Project - $>$ New. Then, the software pops up a dialog for researcher to set the name of the group that will be used to represent the selected HRV.mat files. Actually, the group is created for comparison purposes, which is not necessary for this situation; hence, any name is acceptable. After renaming the group, a file browser dialog appears for the researcher to select HRV.mat files. Once all files are selected, the researcher then clicks Open. Now, on the HRViewer window, the group name appears on the Groups panel, imported HRV.mat filenames appear on the Files panel, and the HRV 
http://wjst.wu.ac.th

indices appear on the HRV Methods panel. Figure 3 presents the front view of the software HRViewer after all 4 files are imported. The researcher is now ready to view HRV.

To view how HRV indices of a subject change over time, the researcher first selects the group of that subject on the Groups panel, then selects the HRV.mat file name of that subject on the Files panel, and finally selects an HRV index on the HRV Methods panel. As a result, the selected HRV time series is plotted on the HRV time series/Statistics panel, the statistics of the HRV measured over the recording is presented as a box plot on the HRV Statistics panel, and the RR interval time series of the selected file is also plotted on the RR interval (s) panel. By changing the HRV indices on the HRV methods panel, it is simple for the researcher to search for HRV indices that correspond to the event under study. Figure 3 shows the output of the software HRViewer when record 103 and MNN index are selected to be viewed.

In addition, the researcher may need to see details of some HRV indices. To do this, the researcher just clicks on the RR interval plot at a time segment. Then, the histogram, power spectrum, Poincaré plot, and DFA plot of the selected segment are presented. Figure 3 shows the details of HRVs measured from the first 5 minutes segment of the RR interval time series. Moreover, if the HRV is computed from the raw ECG file, and the check box Show ECG on the HRViewer window is checked, the raw ECG and R peak detection result of the selected segment will be plotted on the ECGviewer window, as shown in Figure 4 Changing the segment for viewing details of the HRV and ECG signal can be done on the HRViewer window using several methods: by clicking on another segment of the RR intervals panel, or any point on HRV time series/statistics, clicking on the arrow button on the screen, keying the number of segment in the Segment\# text box on the screen, scrolling the mouse wheel, or pressing the left / right arrows on the keyboard. Viewing HRV and ECG in detail may give insightful information about the recording. In addition, in the case of the researcher finding that there is something wrong with a segment, for example, the ECG recording containing severe noises, or the R-peak detection results being invalid, the researcher may remove this segment out of the study by clicking the button Cut on the HRViewer window. The researcher may even remove an HRV.mat file from the group by clicking on the minus button on the Files panel.

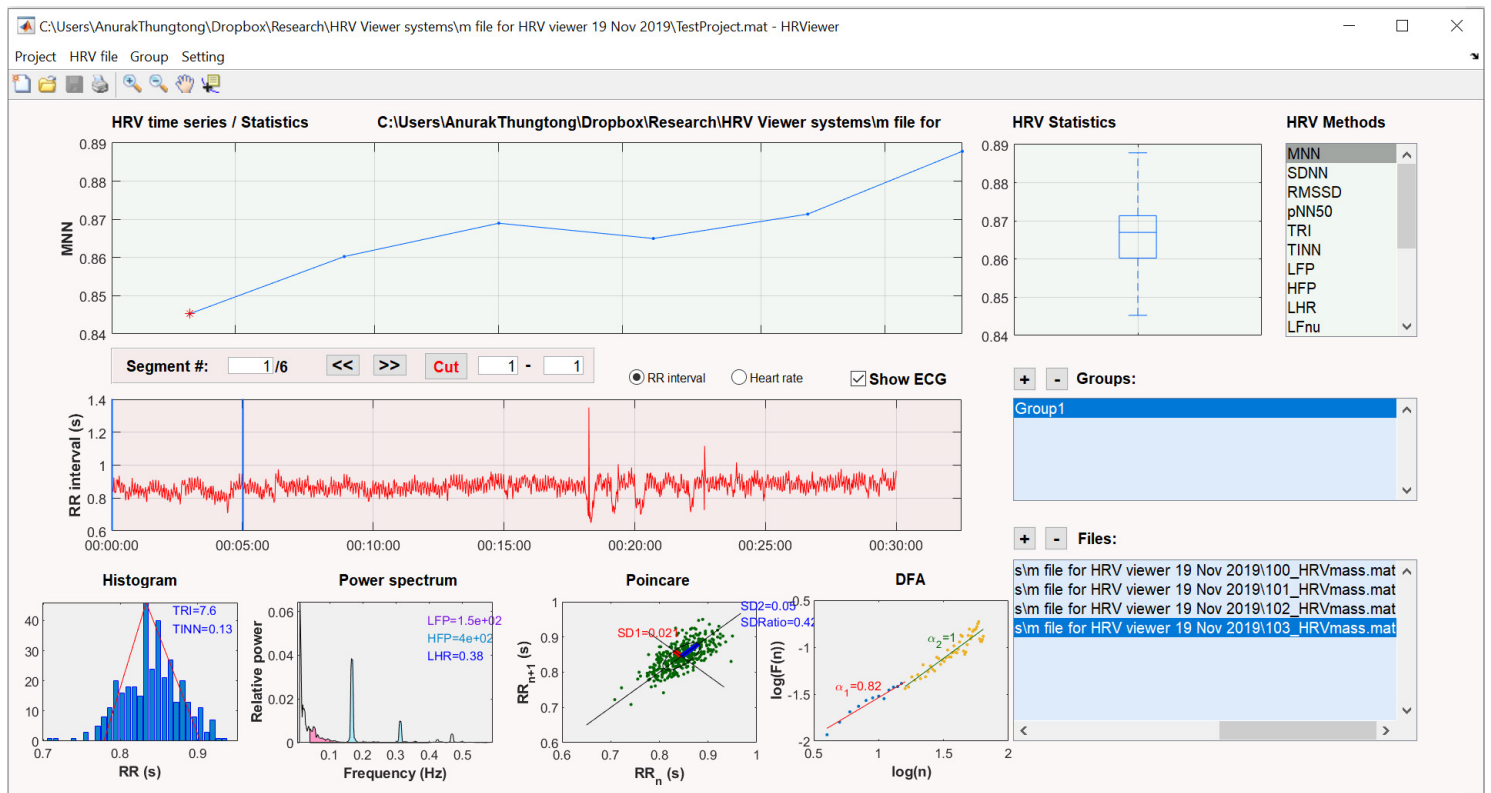

Figure 3 Front view of HRViewer software showing an example of using the software to view how HRV and RR interval time series of a subject changes over time. Details of some HRV indices of a selected RR interval time segment are also presented. 


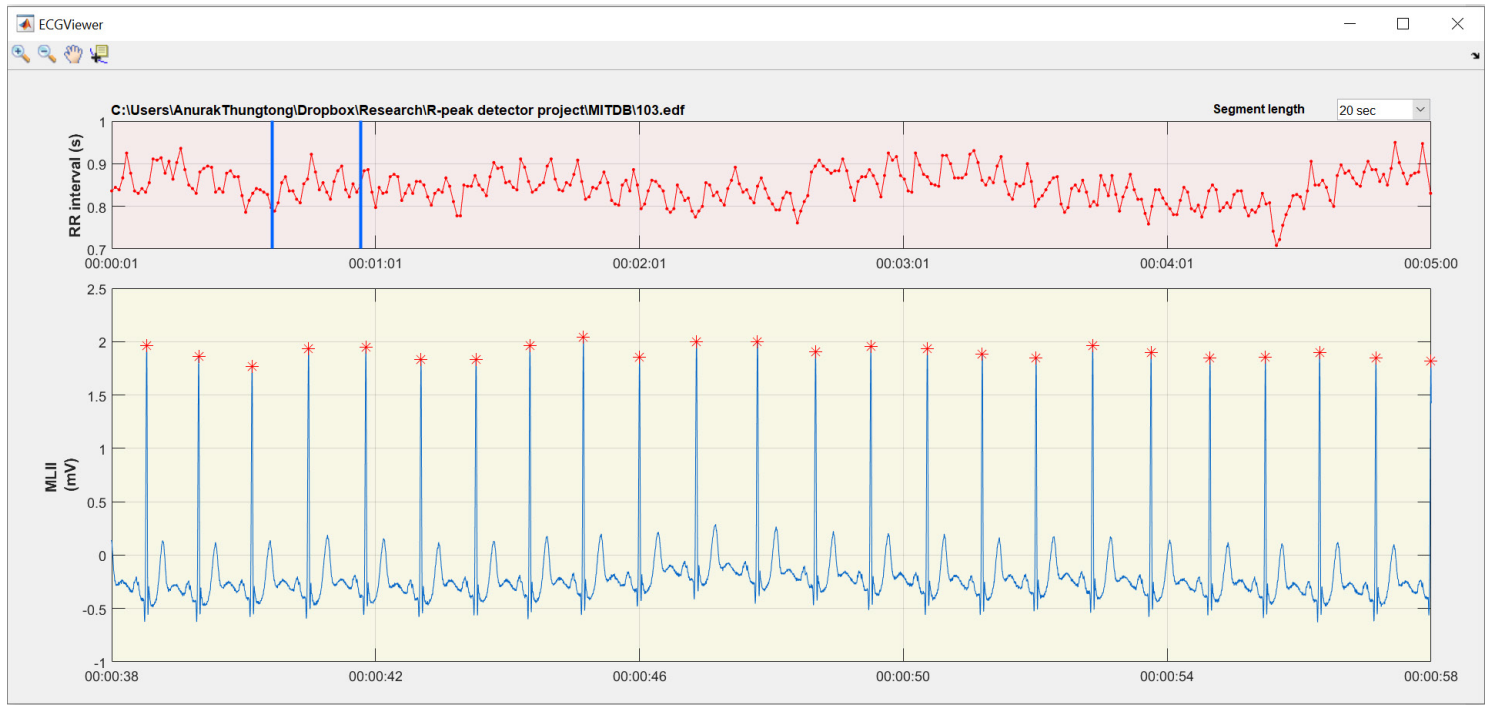

Figure 4 Window for viewing ECG signal and R peak detection results. The RR interval panel of the ECGviewer window is designed as a navigation bar for selecting a segment to view the raw ECG signal and R-peak detection results in even more detail. With both HRViewer and ECGviewer plotting together, the researcher can view the raw ECG signal, R-peak detection results, RR interval time series, detailed HRV indices, HRV time series, and a box plot representing the overall statistics of the HRV time series all at the same time.

\section{Comparing HRV}

Consider another situation in which a researcher would like to study the difference between the HRV of a group of patients with a heart disease and that of a healthy group. Again, the researcher starts from recording the ECG signals from all subjects and computing HRV using the software HRVmass. The software HRVmass produces several HRV.mat output files. These files can be divided into two groups, one belonging to patients with heart disease, another one belonging to the healthy group. For simulation purposes, we employ 13 ECG records from MIT-BIH arrhythmia database as a sample run. Records 100, $104,105,109,112,116$, and 118 are assumed to be the first group, and records 101, 108, 113, 114, 115, and 117 are assumed to be the second group. The HRV.mat files of the first group can be imported to the software HRViewer by clicking on menu Project $->$ New. The researcher then sets the name of the first group to be "Group1" and starts to import the first 7 records to the program. Later, another group and its HRV.mat files can be added to the software by clicking on the menu Group -> Add a new group, and a dialog box appears for naming the second group. After giving a name to the second group of "Group2", the file browser appears for selecting HRV.mat files of the second group. The researcher then selects the last 6 files and clicks Open; now, he is ready to compare HRV.

The comparison starts by selecting both groups on the Groups panel. Then, it can be seen in Figure 5 that the HRV time series/Statistics panel shows a boxplot of the HRV index of each selected file. The panel HRV Statistics displays boxplots of HRV indexes measured from all selected files. Moreover, because the selected files are from different groups, the boxplots on the HRV time series/Statistics have different colors. This is similar to the boxplots in the HRV Statistics, in which one boxplot represents one group. Here, the $p$-value of the MANOVA test of difference between two groups is provided as initial statistical information. The box plots in HRV time series/Statistics and HRV Statistics show that the MNN of both groups are different; hence, MNN can be used as an index for distinguishing patients with heart disease from healthy people. 
http://wjst.wu.ac.th

\section{Conclusions}

In this paper, the HRVmass and HRViewer software for sequential analysis of HRV are introduced. The HRVmass software includes time domain, frequency domain, and non-linear methods for computing HRV in batch processing mode. More HRV methods can be added to the software. The software supports both RR time series and raw ECG signals in EDF format. Although the software has a built-in R peak detection algorithm, it allows users to change the algorithm to a preferred one. In addition, several parameters can be customized using graphical user interface. The HRViewer software shows HRV time series, detailed HRV, such as histogram, power spectra, Poincaré plots, and DFA plots of selected segments. The RR interval time series and ECG signals are also presented. Changing the segment to analyze detailed HRV can be done easily. Graphical comparison of HRV between subjects or groups can be done effectively. With user friendly interfaces, HRVmass and HRViewer allow researchers to easily compute and visualize HRV time series (in terms of both trend and detail), as well as ECG and RR interval time series of large cohort studies.

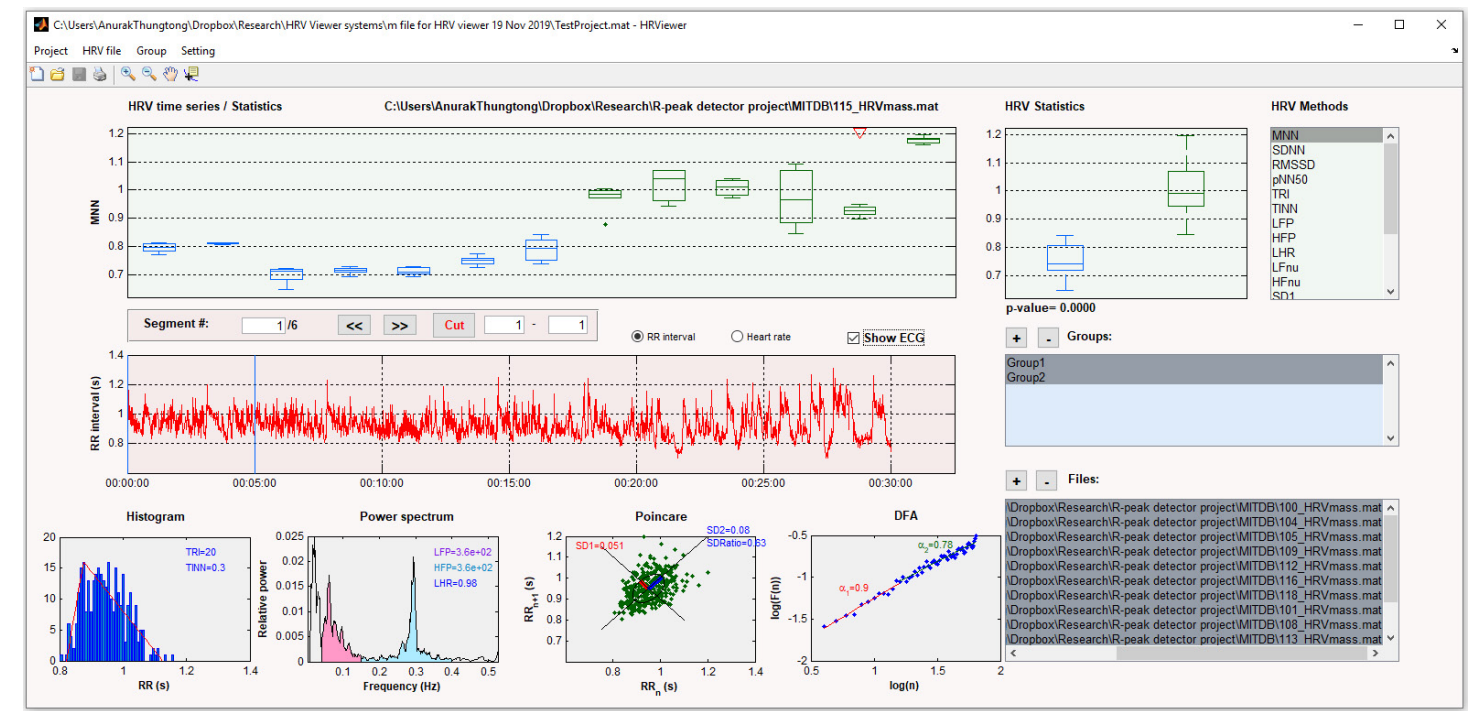

Figure 5 Front view of HRViewer software showing an example of using the software to compare HRV of heart disease and healthy groups. From this simulation, the MNN is the HRV index that differentiates people with heart disease (Group1) from healthy people (Group2).

\section{Acknowledgements}

This research received funding from the National Science and Technology Development Agency, Thailand (project number SCH-NR2016-171 and contract number FDA-CO-2559-3106-TH).

\section{References}

[1] RM Carney, KE Freedland and RC Veith. Depression: The autonomic nervous system, and coronary heart disease. Psychosom. Med. 2005; 67, S29-S33.

[2] DP Zipes. Heart-brain interactions in cardiac arrhythmias: Role of the autonomic nervous system. Cleve. Clin. J. Med. 2008; 75, S94-S96.

[3] UR Acharya, KP Joseph, N Kannathal, CM Lim and JS Suri. Heart rate variability: A review. Med. Biol. Eng. Comput. 2006; 44, 1031-51.

[4] AK Schuster, JE Fischer, JF Thayer, D Mauss and MN Jarczok. Decreased heart rate variability 
http://wjst.wu.ac.th

correlates to increased cardiovascular risk. Int. J. Cardiol. 2016; 203, 728-30.

[5] RM Carney, JA Blumenthal, KE Freedland, PK Stein, WB Howells, Lisa F Berkman, LL Watkins, SM Czajkowski, J Hayano, PP Domitrovich and AS Jaffe. Low heart rate variability and the effect of depression on post-myocardial infarction mortality. Arch. Intern. Med. 2005; 165, 1486-91.

[6] AAS dos Santos, AL Ricci-Vitor, VSR Bragatto, APS dos Santos, EMC Ramos and LCM Vanderlei. Can geometric indices of heart rate variability predict improvement in autonomic modulation after resistance training in chronic obstructive pulmonary disease? Clin. Physiol. Funct. Imag. 2017; 37, 124-30.

[7] R Maestri and GD Pinna. POLYAN: A computer program for polyparametric analysis of cardiorespiratory variability signals. Comput. Meth. Programs Biomed. 1998, 56, 37-48.

[8] JLD Carvalho, FD Rocha, FDO Nascimento, JS Neto and LFJ Junqueira. Development of a Matlab software for analysis of heart rate variability. In: Proceedings of the $6^{\text {th }}$ International Conference on Signal Processing. Beijing, China, 2002, p. 1488-91.

[9] L Rodríguez-Liñares, J Méndez, MJ Lado, DN Olivieri, X Vila and I Gómez-Conde. An open source tool for heart rate variability spectral analysis. Comput. Meth. Programs Biomed. 2010; 103, 39-50.

[10] K Kudryński, P Strumiłło and J Ruta. Computer software tool for heart rate variability (HRV), Twave alternans (TWA) and heart rate turbulence (HRT) analysis from ECGs. Med. Sci. Monit. 2011; 17, MT63-MT71.

[11] P Perakakis, M Joffily, M Taylor, P Guerra and J Vila. KARDIA: A Matlab software for the analysis of cardiac interbeat intervals. Comput. Meth. Programs Biomed. 2010; 98, 83-9.

[12] MP Tarvainen, JP Niskanen, JA Lipponen, PO Ranta-aho and PA Karjalainen. Kubios HRV: Heart rate variability analysis software. Comput. Meth. Programs Biomed. 2014; 113, 210-20.

[13] V Pichot, F Roche, S Celle, JC Barthélémy and F Chouchou. HRV analysis: A free software for analyzing cardiac autonomic activity. Front. Physiol. 2016; 7, 1-15.

[14] B Singh, N Bharti and C Engineering. Software tools for heart rate variability analysis. Int. J. Recent Sci. 2015; 6, 3501-6.

[15] A Thungtong. A robust algorithm for $\mathrm{R}$ peak detection based on optimal Discrete Wavelet Transform. In: Proceedings of the $14^{\text {th }}$ International Joint Conference on Computer Science and Software Engineering. Walailak University, NakhonSiThammarat, Thailand, 2017.

[16] Task Force of the European Society of Cardiology the North American Society of Pacing Electrophysiology. Heart rate variability: Standards of measurement, physiological interpretation and clinical use. Circulation 1996, 93, 1043-65.

[17] WH Press and GB Rybicki. Fast algorithm for spectral analysis of unevenly sampled data. Astrophys. J. 1989, 338, 277-80.

[18] M Brennan, M Palaniswami and P Kamen. Do existing measures of Poincaré plot geometry reflect nonlinear features of heart rate variability? IEEE Trans. Biomed. Eng. 2001; 48, 1342-7.

[19] SM Pincus. Approximate entropy as a measure of system complexity. Proc. Natl. Acad. Sci. 1991; 88, 2297-301.

[20] JS Richman and JR Moorman. Physiological time-series analysis using approximate entropy and sample entropy. Am. J. Physiol. Heart Circ. Physiol. 2000; 278, H2039-H2049.

[21] K Lee. Sample entropy. Available at: https://www.mathworks.com/matlabcentral/fileexchange/ 35784-sample-entropy, accessed April 2020.

[22] CKK Peng, S Havlin, HEE Stanley, ALL Goldberger. Quantification of scaling exponents and crossover phenomena in nonstationary heartbeat time series. Chaos 1995; 5, 82-7.

[23] G Wenye. Detrended Fluctuation Analysis. Available at: https:/www.mathworks.com/ matlabcentral/fileexchange/19795-detrended-fluctuation-analysis, accessed April 2020.

[24] AN Vest, GD Poian, Q Li, C Liu, S Nemati, AJ Shah and GD Clifford. An open source benchmarked toolbox for cardiovascular waveform and interval analysis. Physiol. Meas. 2018; 39, 105004

[25] GB Moody and RG Mark. The impact of the MIT-BIH arrhythmia database. IEEE Eng. Med. Biol. Mag. 2001; 20, 45-50. 